\title{
Immunonutrition in patients with colon cancer
}

\author{
Luis Sánchez-Guillén*, $\neq, 1$ \& Antonio Arroyo**, $\neq, 1$ (iD \\ ${ }^{1}$ Colorectal Unit, Department of General Surgery, Elche University Hospital, Miguel Hernández University, Carrer Almazara, 11, \\ Elche, Alicante 03203, Spain \\ *Author for correspondence: drsanchezguillen@gmail.com \\ **Author for correspondence: arroyocir@hotmail.com \\ ¥ Authors contributed equally
}

“approximately USD $\$ 1000-3000$ was saved per patient treated with preoperative oral supplements, based on the reduction of LOS and the decrease in infectious complications. This supports the use of preoperative immunonutrition, as it seems to be an effective cost measure in patients, resulting in overall reduction of hospital expenses"

First draft submitted: 5 October 2019; Accepted for publication: 18 December 2019; Published online: 6 January 2020

Keywords: colon cancer $\bullet$ immunonutrition $\bullet$ oncologic surgery

\section{Colorectal cancer \& associated complications}

At present, colorectal cancer (CRC) is the third and second most commonly diagnosed cancer in males and females, respectively [1]. In relation to mortality rate, it is ranked second in both sexes. Statistics from the WHO showed that the number of new cases of CRC across the world was 1.8 million in 2018, and 880,792 cases died in the same year [1]. Surgical outcomes in CRC have improved dramatically over the past 50 years due to improvements in preoperative preparation, antibiotic prophylaxis, surgical technique and postoperative management [2]. However, despite the advances in CRC treatment, prevention of complications and postoperative mortality still represents a grey area in the treatment of this disease.

Complications continue to occur such as surgical site infection (SSI), ureteral injury, anastomotic leak, intraabdominal abscess, enteric fistula, bleeding and postoperative bowel obstruction. Postoperative complications significantly affect the short-term outcomes, the length of hospital stay (LOS) and the costs. In addition, it has been demonstrated that postoperative complications after CRC influence the long-term oncological results, along with the patients' quality of life after surgery, playing a crucial role in cancer progression [3,4]. Perioperative morbidity and mortality following colon resection depends, to a large extent, upon whether the procedure is performed under elective or emergency circumstances (e.g., obstructing lesion, bowel perforation), and the patient's associated comorbidities.

Patients with colon cancer are often older and tend to have more comorbidities, and as a result have a higher incidence of postoperative morbidity and mortality [5]. Different studies have evaluated patient outcomes after colorectal surgery and independent risk factors that are associated with an increased risk of in-hospital complications including age, American Society of Anesthesiologists physical status score, hypoalbuminemia, a loss of greater than $10 \%$ total body weight, type and length of operative procedure, intraoperative contamination or low surgeon case volume [6]. Some of them are nonmodifiable risk factors, but others are modifiable for patients who might need specific perioperative optimization and/or proactive measures. Given the frequency of postoperative complications and their implications on quality of life, current work is focusing on the prevention of complications from the preoperative period onwards.

\section{Preparing patients for colorectal surgery}

In the same way someone must prepare for a marathon, patients need to improve their general condition before undergoing surgery in order to avoid/reduce postoperative complications. This strategy, known as prehabilitation, 
includes optimizing nutrition, increasing exercise and mindfulness. These strategies are now also included in the Enhanced Recovery After Surgery protocol [7]. However, unlike in marathons, where few athletes would compete when unfit, most patients undergo surgery when in a worse condition than desired. Malnutrition prevalence has been widely reported to reach $15-40 \%$ in cancer patients at the time of diagnosis, and up to $80-90 \%$ in advanced cases of the disease [8]. The prevalence of malnutrition in CRC patients ranges from 45 to $60 \%$ [9]. It is known that compared with well-nourished patients, malnourished patients are linked to a higher postoperative morbidity and mortality rate, resulting in an up to $50 \%$ increase in LOS and associated health costs, along with a decrease in the perceived quality of life [10]. As a result of this, nutritional perioperative management of patients undergoing elective colorectal surgery has been modified. Surgery is a stressor for patients and can induce changes in both innate and adaptive immune system activity [11]. Major abdominal surgery can cause a release of local and systemic cytokines, inducing a systemic inflammatory response syndrome [12,13]. So, among nutritional interventions, immune-enhancing nutritional supplementation, also referred to as immunonutrition, can be implemented. This is enteral or parenteral supplementation with arginine, glutamine, nonessential fatty acids, branched chain fatty acids, nucleotides or RNA. The use of arginine is based on immune modulatory actions and inflammatory modulation. N-3 fatty acids also modulate the inflammatory response. Nucleotides and RNA supplementation is unlikely to result in a decrease in T-lymphocytes and IL-2 synthesis [14]. The overall objective is to modulate inflammatory responses or enhance protein synthesis after surgery.

The prevalence of surgical infection, especially anastomotic leak and SSIs, is still high despite advances in the management of surgical patients that have emerged in recent years. These infectious complications also result in poor outcomes such as increases in morbidity, LOS, readmission and mortality. In fact, colorectal surgery patients are disproportionately affected by SSI, with rates of up to $25 \%$. Naturally, this is related to the clean-contaminated nature of many colorectal procedures, as well as exteriorization of the bowel; however, up to $55 \%$ of these infections have been deemed preventable [15].

\section{Evidence in favor of preoperative immunonutrition}

Nowadays, there is evidence to support the use of immunonutrition in major digestive oncologic surgery. Although causes of SSI are varied and they are difficult to anticipate, it is not surprising that immunosuppression caused by surgical stress is one of the most important factors associated with its development [16,17]. Several randomized controlled trials and meta-analyses have shown a decrease in overall postoperative complications rate, in particular for infections and a decrease in the LOS. At the same time, different effects were found when compared with all major abdominal surgeries and colorectal surgery. When evaluating all the abdominal surgeries, immunonutrition reduced the risk of overall complications (odds ratio [OR]: 0.79; 95\% CI: 0.66-0.94; 41 trials) and infectious complications (OR: $0.58 ; 95 \%$ CI: $0.51-0.66$; 66 trials), as well as shortened hospital stay (mean difference [MD]: -1.79 days; $95 \%$ CI: -2.39 to $-1.19 ; 52$ trials), but did not seem to alter mortality. Additionally, the existence of bias lowers confidence in the evidence [18]. However, in colorectal surgery, stronger evidence can be found. A randomized controlled trial conducted by our group found a reduction of SSI related to immunonutrient supplements in laparoscopic CRC patients. Patients that received preoperative and postoperative immunonutrition suffered fewer SSIs than those that received dietary advice (11.50 vs $0.00 \% ; \mathrm{p}=0.006)$ [19]. A recent specific meta-analysis for colorectal surgery found significant improvement in the LOS (pooled MD: 2.53; 95\% CI: 1.293.41), infectious complications (pooled OR: 0.33; 95\% CI: 0.21-0.53) that contain the SSIs (pooled OR: 0.25; 95\% CI: 0.22-0.58) and superficial/deep incisional infections (pooled OR: 0.27; 95\% CI 0.12-0.64). Meanwhile, parenteral immunonutrition also improved the immune system (IL-6, CD3, CD4 and CD4/CD8) [20].

Aside from the aforementioned adverse effects on clinical outcome, high infectious complication is linked with significant negative economic consequences. In fact, it is estimated that the USA spends more than $\$ 3$ billion annually on SSIs [15]. Following the evidence related to immunonutrition with lower infectious complications, several studies have evaluated the cost-effectiveness of immunonutrition, comparing the cost and clinical effect versus conventional treatment (nonimmunonutrition). This is the most commonly used technique in pharmacoeconomics, with one requiring criteria from the National Institute for Health and Care Excellence in order to recommend a treatment. They found that approximately USD $\$ 1000-3000$ was saved per patient treated with preoperative oral supplements, based on the reduction of LOS and the decrease in infectious complications. This supports the use of preoperative immunonutrition, as it seems to be an effective cost measure in patients, resulting in overall reduction of hospital expenses [21]. 


\section{Current recommendations}

The benefits of immunonutrition have been widely documented in oncological abdominal surgery, especially in CRC surgery. As a result of this, the European Society for Clinical Nutrition and Metabolism international guidelines, as well as other national guidelines, recommend administration of immunonutrition 7 days before major oncologic surgery in malnourished and high-risk patients [8,22-25]. However, immunonutrition should not be recommended as a routine addition to nutritional supplementation in all surgical patients until higher quality data are obtained. Additional study is required in the future to establish more precise recommendations, adjust specific timings and improve patient selection. At the current time, only surgical patients with malnutrition or healthy individuals undergoing major surgery with an anticipated infectious complication, or lengthy recovery time to return of normal gastrointestinal function, should receive immunonutritional support.

\section{Financial \& competing interests disclosure}

The authors have no relevant affiliations or financial involvement with any organization or entity with a financial interest in or financial conflict with the subject matter or materials discussed in the manuscript. This includes employment, consultancies, honoraria, stock ownership or options, expert testimony, grants or patents received or pending, or royalties.

No writing assistance was utilized in the production of this manuscript.

\section{References}

1. Bray F, Ferlay J, Soerjomataram I, Siegel RL, Torre LA, Jemal A. Global cancer statistics 2018: GLOBOCAN estimates of incidence and mortality worldwide for 36 cancers in 185 countries. CA Cancer J. Clin. 68(6), 394-424 (2018).

2. Paun BC, Cassie S, MacLean AR, Dixon E, Buie WD. Postoperative complications following surgery for rectal cancer. Ann. Surg. 251(5), 807-818 (2010).

3. Merkow RP, Hall BL, Cohen ME et al. Relevance of the C-statistic when evaluating risk-adjustment models in surgery. J. Am. Coll. Surg. 214(5), 822-830 (2012).

4. Vonlanthen R, Slankamenac K, Breitenstein S et al. The impact of complications on costs of major surgical procedures. Ann. Surg. 254(6), 907-913 (2011).

5. 2017 European Society of Coloproctology (ESCP) collaborating group. Safety of primary anastomosis following emergency left sided colorectal resection: an international, multi-centre prospective audit. Color. Dis. 20, 47-57 (2018).

6. Frasson M, Flor-Lorente B, Ramos Rodríguez JL et al. Risk factors for anastomotic leak after colon resection for cancer. Ann. Surg. 262(2), 321-330 (2015).

7. Gustafsson UO, Scott MJ, Schwenk W et al. Guidelines for perioperative care in elective colonic surgery: enhanced recovery after surgery (ERAS $\left.^{\circledR}\right)$ society recommendations. World J. Surg. 37(2), 259-284 (2013).

8. Arends J, Bachmann P, Baracos V et al. ESPEN guidelines on nutrition in cancer patients. Clin. Nutr. 36(1), 11-48 (2017).

9. Klek S, Szybinski P, Szczepanek K. Perioperative immunonutrition in surgical cancer patients: a summary of a decade of research. World J. Surg. 38(4), 803-812 (2014).

10. Schwegler I, von Holzen A, Gutzwiller J-P, Schlumpf R, Mühlebach S, Stanga Z. Nutritional risk is a clinical predictor of postoperative mortality and morbidity in surgery for colorectal cancer. Br. J. Surg. 97(1), 92-97 (2009).

11. Maung AA, Davis KA. Perioperative nutritional support: immunonutrition, probiotics, and anabolic steroids. Surg. Clin. North Am. 92(2), 273-283 (2012).

12. Badia JM, Whawell SA, Scott-Coombes DM, Abel PD, Williamson RCN, Thompson JN. Peritoneal and systemic cytokine response to laparotomy. Br. J. Surg. 83(3), 347-348 (1996).

13. Riese J, Schoolmann S, Beyer A, Denzel C, Hohenberger W, Haupt W. Production of IL-6 and MCP-1 by the human peritoneum in vivo during major abdominal surgery. Shock 14(2), 91-94 (2000).

14. Challine A, Rives-Langes $\mathrm{C}$ et al. Impact of oral immunonutrition on postoperative morbidity in digestive oncologic surgery. Ann. Surg. doi:10.1097/SLA.0000000000003282 (2019) (Epub ahead of print).

15. Turner M, Migaly J. Surgical site infection: the clinical and economic impact. Clin. Colon. Rectal. Surg. 32(03), 157-165 (2019).

16. Faist E, Kupper TS, Baker CC, Chaudry IH, Dwyer J, Baue AE. Depression of cellular immunity after major injury. Arch. Surg. 121(9), 1000-1005 (1986).

17. Piessen G, Muscari F, Rivkine E et al. Prevalence of and risk factors for morbidity after elective left colectomy. Arch. Surg. 146(10), 1149 (2011).

18. Probst P, Ohmann S, Klaiber U et al. Meta-analysis of immunonutrition in major abdominal surgery. Br. J. Surg. 104(12), 1594-1608 (2017). 
19. Moya P, Miranda E, Soriano-Irigaray L et al. Perioperative immunonutrition in normo-nourished patients undergoing laparoscopic colorectal resection. Surg. Endosc. 30(11), 4946-4953 (2016).

20. Xu J, Sun X, Xin Q et al. Effect of immunonutrition on colorectal cancer patients undergoing surgery: a meta-analysis. Int. J. Colorectal Dis. 33(3), 273-283 (2018).

21. Manzanares Campillo M del C, Martín Fernández J, Amo Salas M, Casanova Rituerto D. Estudio prospectivo y randomizado sobre inmunonutrición oral preoperatoria en pacientes intervenidos por cáncer colorrectal: estancia hospitalaria y costos sanitarios. Cir. Cir. 85(5), 393-400 (2017).

22. Calvo Vecino JM, del Valle Hernández E, Ramírez Rodríguez JM et al. Vía Clínica de recuperación intensificada en cirugía abdominal (RICA). (2018). www.grupogerm.es/power/images/viaClinicaRICA.pdf

23. National Institute for Health and Care Excellence. Nutrition support in adults: oral nutrition support, enteral tube feeding and parenteral nutrition (2006). www.nice.org.uk/guidance/cg32/

24. McClave SA, Taylor BE, Martindale RG et al. Guidelines for the provision and assessment of nutrition support therapy in the adult critically Ill patient. J. Parenter. Enter. Nutr. 40(2), 159-211 (2016).

25. Haute Autorite de Sante. Commission Nationale D’Evaluation des Dispositifs Medicaux et des Technologies de Sante: avis de la CNEDiMTS (2016). www.has-sante.fr/upload/docs/application/pdf/2016-03/oral_impact.pdf 